\title{
Sequence variations in RepMP2/3 and RepMP4 elements reveal intragenomic homologous DNA recombination events in Mycoplasma pneumoniae
}

\author{
Emiel B. M. Spuesens, Minoushka Oduber, Theo Hoogenboezem, \\ Marcel Sluijter, Nico G. Hartwig, Annemarie M. C. van Rossum \\ and Cornelis Vink
}

Correspondence

Cornelis Vink

c.vink@erasmusmc.nl

Received 18 February 2009

Revised 25 March 2009

Accepted 30 March 2009
Erasmus MC-Sophia Children's Hospital, Laboratory of Pediatrics, Pediatric Infectious Diseases and Immunity, PO Box 2040, 3000 CA Rotterdam, The Netherlands

\begin{abstract}
The gene encoding major adhesin protein P1 of Mycoplasma pneumoniae, MPN141, contains two DNA sequence stretches, designated RepMP2/3 and RepMP4, which display variation among strains. This variation allows strains to be differentiated into two major P1 genotypes (1 and 2) and several variants. Interestingly, multiple versions of the RepMP2/3 and RepMP4 elements exist at other sites within the bacterial genome. Because these versions are closely related in sequence, but not identical, it has been hypothesized that they have the capacity to recombine with their counterparts within MPN141, and thereby serve as a source of sequence variation of the P1 protein. In order to determine the variation within the RepMP2/3 and RepMP4 elements, both within the bacterial genome and among strains, we analysed the DNA sequences of all RepMP2/3 and RepMP4 elements within the genomes of $23 \mathrm{M}$. pneumoniae strains. Our data demonstrate that: (i) recombination is likely to have occurred between two RepMP2/3 elements in four of the strains, and (ii) all previously described P1 genotypes can be explained by inter-RepMP recombination events. Moreover, the difference between the two major P1 genotypes was reflected in all RepMP elements, such that subtype 1 and 2 strains can be differentiated on the basis of sequence variation in each RepMP element. This implies that subtype 1 and subtype 2 strains represent evolutionarily diverged strain lineages. Finally, a classification scheme is proposed in which the $\mathrm{P} 1$ genotype of $M$. pneumoniae isolates can be described in a sequence-based, universal fashion.
\end{abstract}

\section{INTRODUCTION}

Mycoplasma pneumoniae is a human pathogen from the bacterial class Mollicutes, and represents one of the smallest self-replicating species with respect to both genome size and cellular dimensions (Wilson \& Collier, 1976). The limited size of the genome of this species is generally considered to be the result of a gradual loss of genome information from a common Gram-positive ancestor (Maniloff, 1992). Hitherto, the complete genomic sequence of a single M. pneumoniae strain (M129) has been determined. This sequence has been reported to have a

Abbreviation: SNP, single-nucleotide polymorphism.

The GenBank/EMBL/DDBJ accession numbers for the RepMP2/3 and RepMP4 sequences of the M. pneumoniae strains analysed in this study are FJ603695-FJ604108.

Two supplementary tables, listing sequence identity among the RepMP2/3 and RepMP4 elements from M. pneumoniae strain M129 and GenBank accession numbers of the sequences generated in this study are available with the online version of this paper. length of $816394 \mathrm{bp}$, containing 689 ORFs (Dandekar et al., 2000; Himmelreich et al., 1996). In general, M. pneumoniae is regarded as a genetically highly stable micro-organism. This view is based on the finding of limited sequence diversity between strains as determined by various molecular typing techniques, although this does allow strains to be divided into two major groups (subtype 1 and 2). The molecular discrimination of $M$. pneumoniae isolates in these two subtypes was first described by Dallo et al. (1990) and Su et al. (1990b), who showed the existence of two different sequence variants (subtypes 1 and 2) of the gene encoding the major adhesin of $M$. pneumoniae, i.e. the P1 protein. The sequence differences between these variants have been found to reside within two regions of the P1 (or MPN141) gene ( $\mathrm{Su}$ et al., 1990a). One of these regions, termed RepMP4, is located within the $5^{\prime}$ part of the P1 gene, whereas the other, RepMP2/3, is located within the $3^{\prime}$ part of the gene (Ruland et al., 1990). Interestingly, multiple versions of RepMP4 and RepMP2/3 have been found within the M. pneumoniae genome at sites outside of the P1 gene (Himmelreich et al., 1996; Ruland et al., 1990; Su et al., 1988). In total, eight 


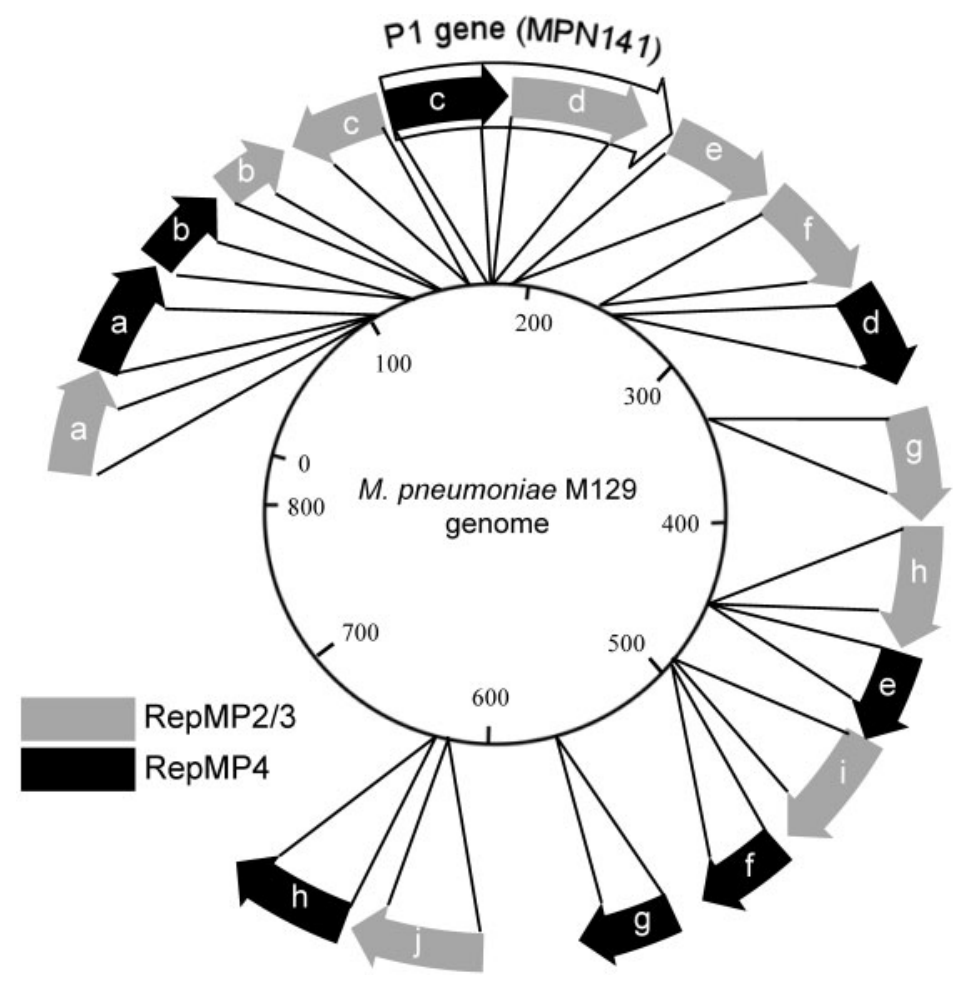

Fig. 1. Structure of the M. pneumoniae MPN141 ORF and distribution of RepMP2/3 and RepMP4 elements within the $M$. pneumoniae genome. The location and orientation of all RepMP2/3 and RepMP4 elements within the M. pneumoniae M129 genome are indicated by grey and black boxes, respectively (Himmelreich et al., 1996). The elements are labelled 'a' to 'j' for RepMP2/3 and ' $a$ ' to ' $h$ ' for RepMP4. The location of the P1 gene is indicated by the arrow at the top. The nucleotide positions of the elements within the genome are listed in Table 2.

versions of the RepMP4 element and 10 versions of the RepMP2/3 element have been found throughout the genome of strain M129 (Fig. 1) (Himmelreich et al., 1996; Ruland et al., 1990). In contrast to the RepMP4 and RepMP2/3 elements, which are situated within MPN141, the other RepMP versions have not been demonstrated to be transcribed or to form part of an operon. The RepMP4 and RepMP2/3 elements have been reported to have lengths of $1.0-1.6 \mathrm{~kb}$ and $0.6-1.9 \mathrm{~kb}$, respectively (Himmelreich et al., 1996). Although the different versions of a given RepMP element are strongly related in sequence, they are not identical (Supplementary Table S1). It was therefore hypothesized that: (i) RepMP elements can rearrange within the M. pneumoniae genome by homologous DNA recombination, and (ii) recombination between the RepMP elements within the P1 gene and those elsewhere in the genome can generate alternative sequence variants of the P1 gene (Rocha \& Blanchard, 2002; Ruland et al., 1990). Obviously, variations within the $\mathrm{P} 1$ gene can result in altered $\mathrm{P} 1$ proteins at the surface of the bacterium. As the $\mathrm{P} 1$ protein is a major immunogen in M. pneumoniae-infected individuals (Hu et al., 1983; Leith et al., 1983; Razin \& Jacobs, 1992; Seto et al., 2005), such events may have a major effect on the virulence and/or spread of M. pneumoniae.
Despite the relatively high intrinsic potential for variation of the P1 gene by means of homologous recombination between the RepMP elements, only a limited number of sequence variants of the P1 gene have currently been identified among isolates of $M$. pneumoniae. In addition, although the characterization of the recombination machinery of $M$. pneumoniae is currently under way (Ingleston et al., 2002; Sluijter et al., 2008), recombinationdriven variation of the P1 gene has thus far not been demonstrated directly. Apart from the two major subtypes (1 and 2) described above, one variant of subtype 1 (variant 1, strain Mp4817) (Dorigo-Zetsma et al., 2001) and two variants of subtype 2 (2a and $2 \mathrm{~b}$ ) (Dorigo-Zetsma et al., 2001; Dumke et al., 2006; Kenri et al., 1999) have been reported. Strain Mp4817 has been shown to deviate from regular subtype 1 strains in having a significantly different DNA sequence within part of the RepMP2/3 element of the P1 gene (Dorigo-Zetsma et al., 2001). Likewise, the 2a and $2 \mathrm{~b}$ variants have been demonstrated to differ from regular subtype 2 strains in a relatively small sequence within the $3^{\prime}$ half of the RepMP2/3 element of the P1 gene (DorigoZetsma et al., 2001; Dumke et al., 2006; Kenri et al., 1999). In addition, a single isolate (Mp3896) has been identified that cannot be classified as a regular subtype 1 or 2 strain, 
Table 1. Names and P1 genotypes of the $M$. pneumoniae strains used in this study

\begin{tabular}{|llc|}
\hline Number & \multicolumn{1}{c|}{ Strain } & P1 genotype \\
\hline 1 & M129 (ATCC 29342) & 1 \\
2 & MAC (ATCC 15492) & 2 \\
3 & P1 1428 (ATCC 29085) & 1 \\
4 & Mp5 & 1 \\
5 & Mp22 & 1 \\
6 & Mp72 & $1^{*}$ \\
7 & Mp1042 & $1^{*}$ \\
8 & Mp1116 & 1 \\
9 & Mp1286 & $1^{\star}$ \\
10 & Mp1397 & $1^{*}$ \\
11 & Mp1842 & 2 \\
12 & Mp2004 & $2^{*}$ \\
13 & Mp2018 & $1^{*}$ \\
14 & Mp2157 & $1^{*}$ \\
15 & Mp4599 & $2^{*}$ \\
16 & Mp4817 & $2^{*}$ \\
17 & Mp5181 & $1^{*}$ \\
18 & Mp5191 & $1^{*}$ \\
19 & Mp5192 & 2 \\
20 & Mp5194 & $2^{*}$ \\
21 & Mp5196 & $1^{*}$ \\
22 & Mp5245 & $2^{*}$ \\
23 & Ofo & \\
& & \\
\hline
\end{tabular}

${ }^{\star}$ Determined in this study. The genotypes of the other strains have been described before (Dorigo-Zetsma et al., 2000).

and which differs from all other known strains in both the RepMP4 and the RepMP2/3 elements of its P1 gene (Pereyre et al., 2007).

Many questions remain as to the origin of the variation within the P1 gene of $M$. pneumoniae strains, including the strains mentioned above. There are two main reasons for this: (i) the (variations in) RepMP sequences both within and outside of the P1 gene have not yet been studied systematically, and (ii) only a single $M$. pneumoniae genome sequence is currently available. Nevertheless, a study on a variant 2a strain (strain 309) by Kenri et al. (1999) suggested that inter-RepMP2/3 element recombination had occurred in this strain. This was based on the similarity between the variant $2 \mathrm{a}$-specific sequence within the P1 gene and a RepMP2/3 element elsewhere in the genome (Kenri et al., 1999). Homologous recombination events have also been suggested to underlie the atypical sequences of the P1 gene of strains Mp3896 and Mp4817, although the RepMP2/3 and RepMP4 elements outside of the P1 gene in these strains have not been analysed (Dorigo-Zetsma et al., 2001; Pereyre et al., 2007). Interestingly, it has been reported that strain Mp4817 contains a hybrid P1 sequence that possesses both subtype 1 - and subtype 2-specific sequences (Dorigo-Zetsma et al., 2001).
In order to increase our understanding of the occurrence as well as the dynamics of intragenomic recombination of RepMP2/3 and RepMP4 elements in M. pneumoniae, and to shed light on the origins of the subtype 1 and 2 differences among strains, we have determined the sequences of all 10 RepMP2/3 and all eight RepMP4 elements in a set of 23 different M. pneumoniae isolates. Our data show that the occurrence of intragenomic recombination of RepMP $2 / 3$ elements is a relatively common phenomenon in $M$. pneumoniae and that all RepMP2/3 and RepMP4 elements carry either a subtype 1or a subtype 2-specific signature sequence. As a consequence, the $M$. pneumoniae genome possesses either subtype 1- or subtype 2-specific sequences, but not both, indicating that these subtypes represent evolutionarily separated strain lineages.

\section{METHODS}

Strains. We used 23 different M. pneumoniae strains as listed in Table 1. These strains have been described by Dorigo-Zetsma and co-workers (Dorigo-Zetsma et al., 2000, 2001), and were kindly provided by Dr S. A. Zaat (Academic Medical Center, Amsterdam). Since the complete genome sequence is currently only available for strain M129 (ATCC 29342; a subtype 1 strain), we used this strain as a reference for the sequences of the RepMP2/3 and RepMP4 elements (Dandekar et al., 2000; Himmelreich et al., 1996). The P1 gene sequence, however, is available for a few other strains. Of these, we used PI 1428 (ATCC 29085) and Mp22 as representatives of subtype 1 strains. Strain Mp4817 was employed as the prototype 'variant 1' strain, whereas MAC (ATCC 15492) and Mp1842 were regarded as subtype 2 strains.

M. pneumoniae cultures. All strains were cultured in Mycoplasma medium containing $1.4 \%$ Difco PPLO broth (Becton Dickinson), $0.15 \%$ Difco TC Yeastolate, UF (Becton Dickinson), $1.4 \%$ glucose, $20 \%$ horse serum, $1000 \mathrm{U}$ penicillin $\mathrm{G} \mathrm{ml}^{-1}, 500 \mathrm{U}$ polymyxin $\mathrm{B}$ $\mathrm{ml}^{-1}$ and $0.02 \mathrm{mg}$ phenol red $\mathrm{ml}^{-1}$. The $\mathrm{pH}$ of the medium was adjusted to 7.8-8.0 using $2 \mathrm{M} \mathrm{NaOH}$, followed by filter-sterilization. Strains were grown in $3 \mathrm{ml}$ medium at $37{ }^{\circ} \mathrm{C} / 5 \% \mathrm{CO}_{2}$ in $25 \mathrm{~cm}^{2}$ tissue-culture flasks (Greiner). Cells were harvested upon colour change of the medium (from red/orange to yellow).

Genomic DNA purification. Genomic DNA was isolated from $M$. pneumoniae cells as follows. Cultures $(3 \mathrm{ml})$ were harvested by centrifugation for $10 \mathrm{~min}$ in a microcentrifuge at full speed. The cell pellet was resuspended and washed in $1 \mathrm{ml} 0.9 \% \mathrm{NaCl}$, followed by centrifugation. The resulting pellet was resuspended in $400 \mu \mathrm{l} \mathrm{TE}$ buffer (10 mM Tris/HCl, $1 \mathrm{mM}$ EDTA, pH 8.0). Then, $70 \mu \mathrm{l} 10 \%$ SDS and $5 \mu \mathrm{l}$ proteinase $\mathrm{K}\left(10 \mathrm{mg} \mathrm{ml} \mathrm{m}^{-1}\right)$ were added. After incubation for $10 \mathrm{~min}$ at $65{ }^{\circ} \mathrm{C}, 100 \mu \mathrm{l} 5 \mathrm{M} \mathrm{NaCl}$ was added, followed by the addition of $100 \mu 10 \% N$-cetyl- $N, N, N$-trimethylammonium bromide (CTAB) in $0.7 \mathrm{M} \mathrm{NaCl}$. The solution was incubated for $10 \mathrm{~min}$ at $65{ }^{\circ} \mathrm{C}$, after which the DNA was extracted from the solution using $500 \mu \mathrm{l}$ chloroform : isoamyl alcohol (24:1; $\mathrm{v} / \mathrm{v})$. The DNA-containing aqueous phase was transferred to a fresh tube and the DNA was precipitated by the addition of $360 \mu \mathrm{l} 2$ propanol. Following incubation at $-20{ }^{\circ} \mathrm{C}$ for $30 \mathrm{~min}$, the DNA was pelleted by centrifugation at $16000 \mathrm{~g}$ for $10 \mathrm{~min}$ in a microcentrifuge. After a wash step with $70 \%$ ethanol, the DNA was dried, and resuspended in $10 \mu \mathrm{l} \mathrm{H}_{2} \mathrm{O}$.

Amplification and cloning of RepMP2/3 and RepMP4 elements. In order to sequence all 10 RepMP2/3 and all eight RepMP4 
Table 2. Names and genome locations of the RepMP2/3 and RepMP4 elements and sequences of primers used for amplification of the elements

\begin{tabular}{|c|c|c|c|c|}
\hline Element ${ }^{\star}$ & Genome position $\dagger$ & $\begin{array}{c}\text { Element length } \\
\text { (bp) }\end{array}$ & $\begin{array}{l}\text { Primer } 1 \text { sequence } \\
\qquad\left(5^{\prime}-3^{\prime}\right)\end{array}$ & $\begin{array}{c}\text { Primer } 2 \text { sequence } \\
\qquad\left(5^{\prime}-3^{\prime}\right)\end{array}$ \\
\hline RepMP2/3-a & $127355-128821$ & 1467 & ttccgtgctaacttaggttc & aaatggaggtagagcgaattg \\
\hline RepMP2/3-c & $169920-171370$ & 1451 & gggaattggtttgaccggag & gcacttgaaagagttagttgat \\
\hline RepMP2/3-d & $182860-184769$ & 1910 & gtccaatccaaaatgtggttg & actactgaggttaccactactcg \\
\hline RepMP2/3-e & $190154-191782$ & 1629 & taagagagaagtgatgcagc & caccactaagcacactgccaat \\
\hline RepMP2/3-g & $341759-343166$ & 1408 & gaacccgcatccactttttc & cgtcccaaataccggtgtga \\
\hline RepMP2/3-h & $441236-442915$ & 1680 & ggcacttgaaagagttagttaac & tgccaatgttaccactattcg \\
\hline RepMP2/3-i & $492620-494206$ & 1587 & ttgaacaacaattaggtcaag & gtgtcgttgttttgagcgac \\
\hline RepMP2/3-j & $606031-607756$ & 1726 & cctagtgtggcgaaaatcag & ttgacctgagcctgaagaac \\
\hline RepMP4-a & $130355-131994$ & 1640 & tcccactaaataaattgagttg & atcagtggtggtattggaatc \\
\hline RepMP4-b & $165770-167046$ & 1277 & acaactattagtagtagaagac & caacttactgtttaaaagttgc \\
\hline RepMP4-g & $565050-566094$ & 1045 & cctaacgacaagaataagtac & gtgaggtaattaatccatagac \\
\hline RepMP4-h & $609094-610639$ & 1564 & tcccactaaataaattgagttg & gcgccaccaacttggtatcc \\
\hline
\end{tabular}

${ }^{*}$ The RepMP2/3 and RepMP4 elements were individually named with the suffixes -a to $-\mathrm{j}$ and $-\mathrm{a}$ to $-\mathrm{h}$, respectively.

$\dagger$ The genome positions and sequences were derived from GenBank file U00089.

elements from each strain, 18 specific PCR primer sets were designed based on the genome sequence of M. pneumoniae strain M129 (GenBank accession no. U00089). The sequences of the primers, which were synthesized by and purchased from Eurogentec, are listed in Table 2. For all strains, purified chromosomal DNA was used to amplify each RepMP2/3 and RepMP4 element by high-fidelity PCR. The PCR mixture $(25 \mu \mathrm{l})$ contained either 0.5 or $1.0 \mu \mathrm{M}$ primer 1 , either 0.5 or $1.0 \mu \mathrm{M}$ primer $2,0.2 \mathrm{mM}$ of each dNTP (Fermentas), $0.02 \mathrm{U} \mathrm{\mu l}^{-1} \quad P f u$ DNA polymerase (Fermentas), $1 \times P f u$ buffer containing $\mathrm{MgSO}_{4}$ (Fermentas) and $10 \mathrm{ng}$ purified M. pneumoniae DNA. For the majority of the PCRs, the following conditions were used: $5 \mathrm{~min}$ at $94{ }^{\circ} \mathrm{C}$, followed by 35 cycles of $30 \mathrm{~s}$ at $94{ }^{\circ} \mathrm{C}$, $30 \mathrm{~s}$ at $52{ }^{\circ} \mathrm{C}$, and $4 \mathrm{~min}$ at $72{ }^{\circ} \mathrm{C}$. For amplification of the RepMP2/3-h element, the annealing temperature was lowered to $45^{\circ} \mathrm{C}$. The resulting PCR fragments, of which the lengths are listed in Table 2, were purified by ethanol precipitation and cloned into HincIIdigested vector pBluescript $\mathrm{SK}^{-}$(Stratagene). In some cases, purified PCR products were used directly in DNA sequencing reactions (see below).

Sequencing of RepMP2/3 and RepMP4 elements. Automated DNA sequencing was performed using ABI PRISM BigDye Terminator (BDT) Cycle Sequencing Ready Reaction kits and a $3130 x l$ Genetic Analyzer (Applied Biosystems). Sequencing reactions $(10 \mu \mathrm{l})$ contained $1 \mu \mathrm{l}$ BDT Ready Reaction premix (Applied Biosystems), $5 \times$ sequencing buffer (Applied Biosystems), $3.2 \mu \mathrm{M}$ of either M13 reverse or M13 forward primer (Eurogentec), and $1 \mu \mathrm{l}$ plasmid DNA or PCR product (150-300 ng). Since most of the RepMP elements are larger than $1 \mathrm{~kb}$, new primers were designed in order to close all sequences double-stranded using a 'primer-walking' strategy. Raw DNA sequence data were processed using Chromas version 2.33 software (Technelysium) and assembled using the application SeqMan II (DNASTAR).
The GenBank accession numbers (http://www.ncbi.nlm.nih.gov/) of the nucleotide sequences generated in this study are listed in Supplementary Table S2.

\section{RESULTS}

\section{Amplification and sequencing of RepMP2/3 and RepMP4 elements}

In order to determine the nucleotide sequences of all RepMP2/3 and RepMP4 elements of the 23 M. pneumoniae strains, we designed 18 element-specific PCR primer sets (Table 2). The sequences of the primers were derived from the genome sequence of strain M129, and were chosen so as to include the complete RepMP2/3 and RepMP4 elements as defined by Himmelreich et al. (1996). Each primer set gave rise to specific PCR products for each of the 23 strains. Thus, a total of 414 PCR products were generated that were each cloned into the E. coli plasmid pBluescript $\mathrm{SK}^{-}$and sequenced. If unique sequence differences were found between the congruent elements from different strains, these differences were confirmed by resequencing (using newly amplified DNA fragments). In each of these cases, we could confirm the previously observed differences. As a consequence, we did not detect any mutations induced by PCR amplification. We did find several examples of putative sequence errors, however, in previously deposited GenBank files; these errors are listed in the legends to Figs $2-4$. 
In order to distinguish between the 10 RepMP2/3 and eight RepMP4 elements, we employed a novel systematic nomenclature for the elements (Fig. 1, Table 2) that is based on their appearance within the genome and the current numbering of the M. pneumoniae M129 genome in the GenBank database. This novel nomenclature was necessary because the current genome numbering differs from that originally reported by Himmelreich et al. (1996). Previously, the elements were not named systematically in the literature, and some elements have been indicated by a number (Kenri et al., 1999). In order to avoid confusion with this previous numbering, we have proposed a nomenclature that includes the suffixes $-\mathrm{a}$ to $-\mathrm{j}$ for the 10 RepMP2/3 elements and -a to $-h$ for the eight RepMP4 elements, as listed in Table 2.

\section{Sequence variation within RepMP2/3 elements among $M$. pneumoniae strains}

The sequences of the RepMP2/3 elements are shown in Fig. 2. One of the most significant findings from the analysis of these sequences is that each element possesses either a subtype 1 signature (the nucleotides indicated in blue in Fig. 2) or a subtype 2 signature (the nucleotides indicated in red). Thus, a subtype 1 strain exclusively contains subtype 1-specific RepMP2/3 elements and a subtype 2 strain only contains subtype 2 -specific RepMP2/3 elements. The differences between these subtypes are defined by single-nucleotide polymorphisms (SNPs) (in each element in Fig. 2), by deletions of one nucleotide (twice in RepMP2/3-f) or three nucleotides (in RepMP2/3-a and RepMP2/3-j), as well as by an insertion of six nucleotides (in RepMP2/3-e). Almost all elements also differ among strains in a fashion that is independent of the subtype to which they belong. In most cases, these 'subtype-independent' differences are SNPs. The highest level of variation among strains was observed in two of the RepMP2/3 elements, i.e. in RepMP2/3-d, which is located within the MPN141 gene (Fig. 3), and in RepMP2/3-c (Fig. 2c). Most of the nucleotide sequence differences within element RepMP2/3-d are subtype-specific. As described above, these differences have previously been employed for the classification of strains as subtypes 1, 2, 2a and 2b. It has been suggested that the RepMP2/3-d element from subtype 2a strain 309 contains sequences that are derived from another RepMP2/3 element, i.e. RepMP2/3-a (Kenri et al., 1999). Indeed, we also found the sequence between positions 184491 and 184751 within RepMP2/3-d from subtype $2 \mathrm{a}$ strains to be identical to a sequence within RepMP2/3-a; this sequence is identical in subtype 1 and 2 strains (Fig. 3). Interestingly, the availability of the sequences of all RepMP2/3 elements from both subtype 1 and 2 strains enabled the tracing of the putative origin of 'aberrant' sequences within the MPN141 gene of the other variant strains, i.e. strains Mp4817 and Mp3896, and 2b strains. As shown in Fig. 3, the variable part of the RepMP2/3-d element in strain Mp4817 is identical, except for a single-nucleotide substitution, to sequences from element RepMP2/3-e as found in all subtype 1 strains, including Mp4817. Consequently, in contrast to what has been suggested (Dorigo-Zetsma et al., 2001), the RepMP2/ 3-d element from Mp4817 exclusively contains subtype 1specific sequences and is not a hybrid of subtype 1- and subtype 2-specific sequences. Within strain Mp3896, the RepMP2/3-d element contains sequences that are identical to those of two other RepMP2/3 elements, i.e. RepMP2/3-g from subtype 2 strains (between positions 183508 and 183852 ) and RepMP2/3-a (between positions 184491 and 184751 , as found in subtype 2a strains) (Fig. 3). Finally, subtype $2 \mathrm{~b}$ strains contain a relatively large sequence within their RepMP2/3-d elements (from position 184194 to 184704) that is identical to a sequence from element RepMP2/3-a as found in all investigated subtype 2 strains (Fig. 2a).

The second highly variable RepMP2/3 element within our strain collection was RepMP2/3-c. In four of the strains, i.e. Mp5, Mp72, Mp2157 and Mp5181, this element contains a $1.5 \mathrm{~kb}$ sequence that is identical to the sequence of RepMP2/3-h from the same strain, except for four singlenucleotide deletions in both Mp72 and Mp5181, and two single-nucleotide deletions in Mp2157. As a consequence, these strains carry two RepMP2/3-h sequences, one of which replaces the RepMP2/3-c sequence typically found in other strains. These data strongly support the hypothesis that RepMP2/3 elements can recombine in a nonreciprocal, unidirectional fashion. Furthermore, these results show that recombination between these elements can occur at genome sites other than MPN141. It is noteworthy that the putative recombination event involving RepMP2/3-c and RepMP2/3-h has taken place independently in four different strains. This is inferred from the presence of differences between these strains in their RepMP2/3-h sequences, which are also copied to their putative second location at the RepMP2/3-c locus. Moreover, these four strains include three subtype 1 strains $(\mathrm{Mp} 5, \mathrm{Mp} 72$ and Mp2157) and one subtype 2 strain (Mp5181), indicating that this DNA rearrangement is not linked exclusively to a specific subtype.

\section{Sequence variation within RepMP4 elements among $M$. pneumoniae strains}

The sequences of the eight RepMP4 elements from the 23 strains are summarized in Fig. 4. As was found for the RepMP2/3 elements, each RepMP4 element possesses either a subtype 1 signature (the nucleotides indicated in blue in Fig. 4) or a subtype 2 signature (the nucleotides indicated in red). The differences between the two subtypes are principally defined by SNPs (in each RepMP4 element), but also by deletions of one and 12 nucleotides (both in RepMP4-a) and by insertions of three and 72 nucleotides (both in RepMP4-d). We found these 72 nucleotides to also be present in two other elements of subtype 2 strains, i.e. in RepMP4-a [between positions 131502 and 131 573, with a single mismatch at position $131522(\mathrm{C}>\mathrm{G})$ ] and in 
(a)

RepMP2/3-a 127355-128821

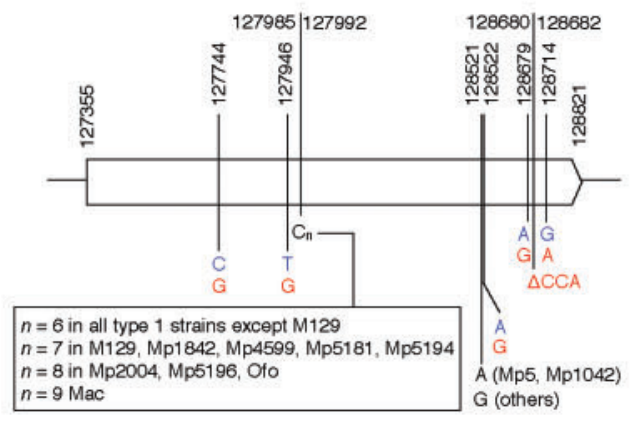

(c)

RepMP2/3-c 169920-171370

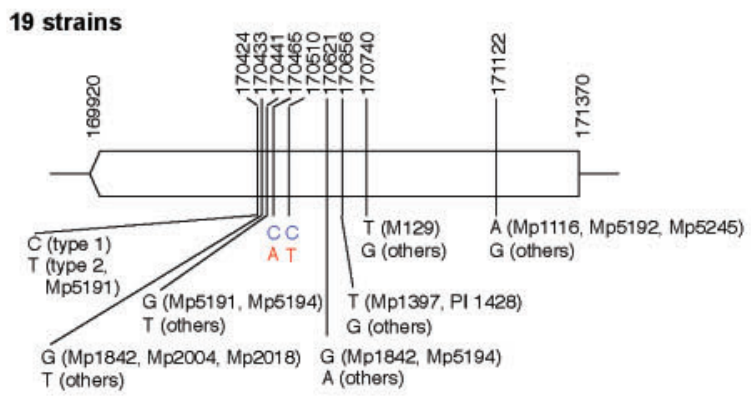

(b)

RepMP2/3-b 168051-168681

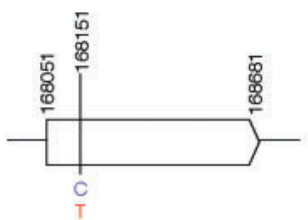

(d) $\begin{gathered}\text { RepMP2/3-d } \\ 182860-184769\end{gathered}$

4 strains: Mp5, Mp72, Mp2157, Mp5181:

Sequence identical to RepMP2/3-h (441237 - 442786)

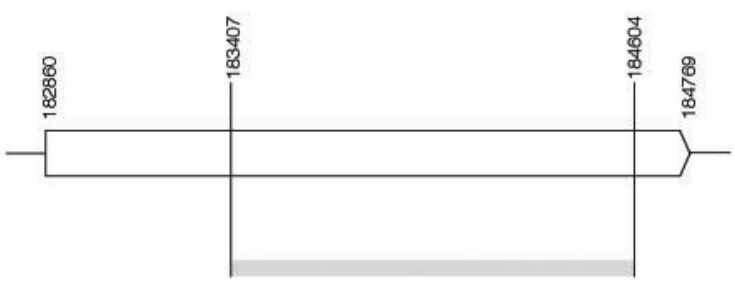

Variable region between subtype 1 and subtype 2 strains (see Fig. 3)

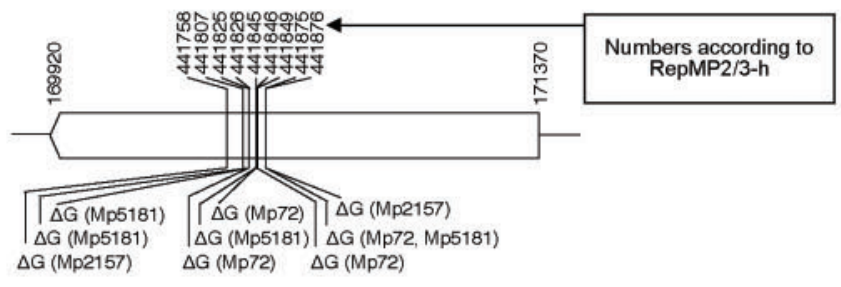

(e)

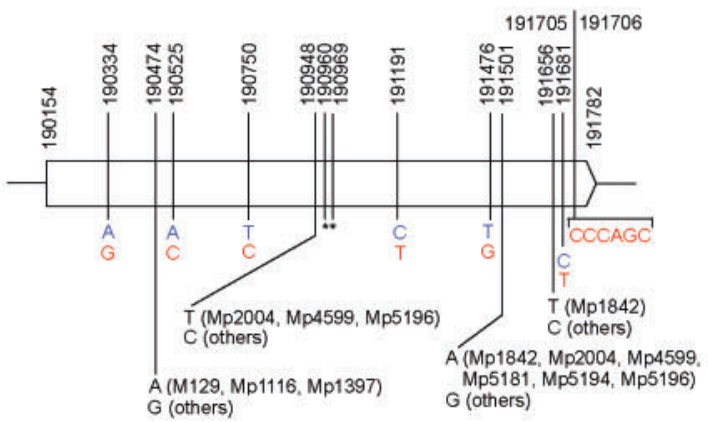

(f)

RepMP2/3-f

245562-247243

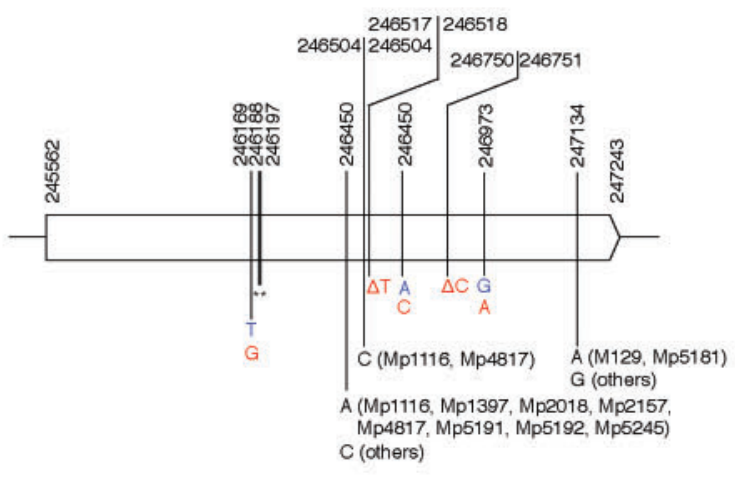


(g)

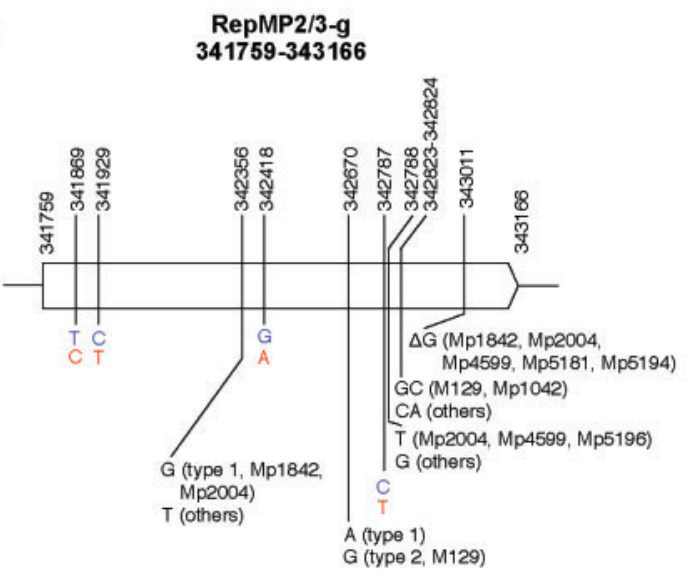

(i)

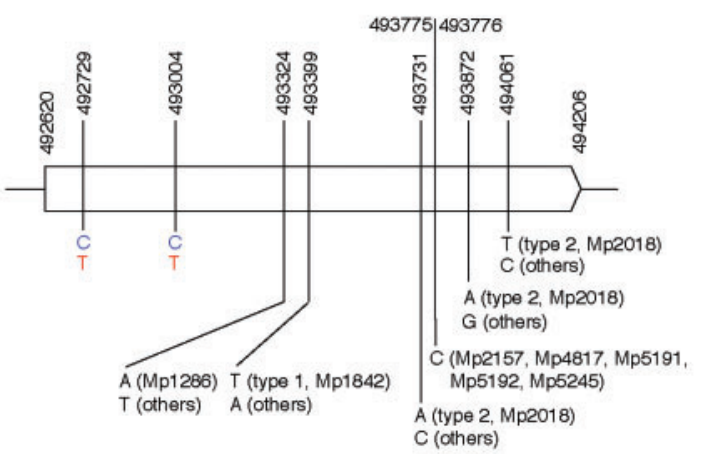

(h)

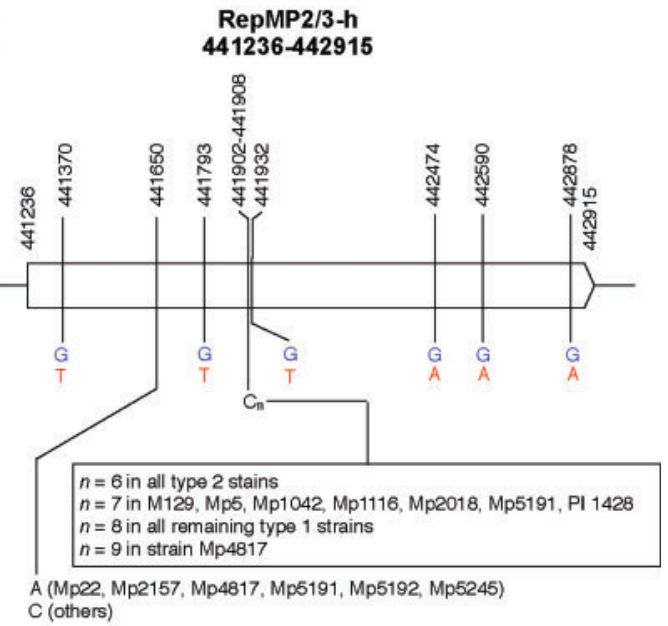

(j)

RepMP2/3-j $606031-607756$

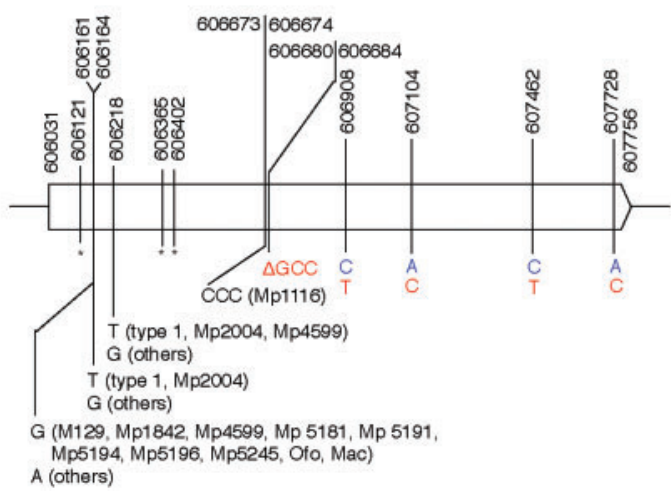

Fig. 2. Sequence variation among the $10 \mathrm{RepMP} 2 / 3$ elements within the $23 \mathrm{M}$. pneumoniae strains. The sequences of the elements RepMP2/3-a to RepMP2/3-j are schematically represented in (a-j). The boxes indicate the length (drawn to scale) and orientation of each element and only list those nucleotide sequences within a given element that differ among the strains or between any strain and sequenced strain M129 (Himmelreich et al., 1996). Below the name of each element, its position within the genome of M129 is listed, as derived from GenBank accession number U00089. The other positions in the figure also refer to this sequence. Nucleotides, insertions and deletions $(\Delta)$ that are found in all subtype 1 and all subtype 2 strains are indicated in blue and red, respectively. Sequence variations that are not subtype-specific are indicated in black. At some positions, indicated with an asterisk, the M129 sequence from GenBank differed from the sequences of M129 and all other strains as determined in this study. We therefore assume that the GenBank file is not correct at these positions. The incorrect nucleotides are: in RepMP2/3-e, a T at position 190960 (should be a C) and a G at position 190969 (should be an A); in RepMP2/3-f, a T at position 246188 (should be a C) and a T at position 246197 (should be a C); in RepMP2/3-j, a T at position 606121 (should be a C), an A at position 606365 (should be a T) and a C at position 606402 (should be a G). Since element RepMP2/3-d, which is located within the P1 gene, is highly variable, the sequence variations within this element are depicted in a separate figure (Fig. 3 ).

RepMP4-g [between positions 565811 and 565852 , with a mismatch at position $565840(\mathrm{C}>\mathrm{A})]$. In addition to these subtype-specific differences, most of the elements also display subtype-independent SNPs, albeit to a far lesser extent than do the RepMP2/3 elements. The element that shows the highest level of sequence variability is the one located within the MPN141 ORF, i.e. RepMP4-c (Fig. 5). The variations within this element are numerous and are 


\title{
RepMP2/3-d \\ $180820-182441$
}

\begin{abstract}
183587 (1) AAACCACACCAAGTTCACGAGCGCTACGGGGATGGACCAGCAGGGACAATCAGGTACCTCCGCGGGGAATCCCGACTCGTTAAAGCAGGATAATATTAGTAAGAGTGGGGATAGTTTAACCACGCAGGACGGCAATG (2) ---CCACACCAAGTTCACGAGCGCTACGGGGATGGGCCAGCAGGAACAATCAGGTACCTCCGCGGGGAATCCCGACTCGTTAAAGCAGGGATAAGATTAGTAAGAGTGGGGATAGGTTTAACCACGCAGGACGGCAATT

183724 (1) CGATCGATCAACAAGAOGGCACCAACTACACCAACCTCCCCCCCAACCTCACCCCCACCGCTGATTGACCGAAC $O C G C T G T C A T T C A C C A A C A A G A A C A A C G C G C A G C G C G C C C A G C T C T T C C T C C O C G O C T T G T T G$ (2) CGATGGATCAACAAGAGGCCACCAACTACACCAACCTCCCCCCCAACCTCACCCCCACCGCTGATTGACCGAACGCGCTGTCATTCACCAACAAGAACAACGCGCAGCGCGCCCAGCTGTTCCTGCGCGGCCTGTTG A (Mp1842, Mp5194)

183861 (1) GGCAGCATCCCGGTGTTGGTGAATCGAAGTGGGTCCGATTCCAACA---AATTCCAAGCCACOGACCAAAAATGGTCCTACACCGACTTACATTCGGACCAAACCAAACTGAACCTCCCCGCTTTACGGTGAGGTGAA (2) GGCAGCATCCCGGTGTTGGTIAATAAGTCCOGCCAAGATGATAACAGTAAGTTTAAGGCGGAGGACCAAAAATGGTCCTACACCGACTTACAGTCGGACCAAACCAAACT GAACCTCCCCGCTTACGGTGAGGTGAA

183998 (1) TGGGTTGTTGAATCCGCCGTTGGTGGAAACCTATTTTGGGAACACGCGAGCGGGTGGTTCGGGGTCCAACACGACCAGTTCACCCGGTATCGGTTTTAAAATTCCCGAACAAAATA--------ATEAT-TCCAAAG (2) TGGGTTGTTGAATCCGCCGTTGGTGGAAACCTATTTTGGGAACACGCGAGCGAGTGGTTCGGGGTCCAACACGACCAGTTCACOCGGTATCGGTTTTPAAATTCCCGAACAAAGTGGCACAAACACAACGTCGAAGG

184135 (1) CCACCCTGATCACCCCCGGGTTGOCTTGAACGCCOCAGGACGTCGGTAACCTCGTTGTCAGT GSCACCACOGTEAGCTTCCAGCTCGOCGGGTGGCTGGTCACCTTCACGGACTTTGTCAAACCCCCGCGCOGGTTAC (2) CTGTGCTGATCACCCCCGGGTTGGCTTGAACGCCOCAAGACGTTGGTAACCTCGTTGTCAGTGGCACCAGCTTCAGCTTCCAGCTCGGCGGGTGGITAGTIACGTTCACGGACTTTATCAAACCCCGCGCTGGTTAC

184272 (1) CTCGGTCTCCAGTTAACGGGCTTGGATGCAAGTGATGCGACGCAGCGCGCC

(2) CTCGGGCTCCAGTTAACGGGCTTGGATGCAAGTGATGCGACGCAGCGCGCT$$
\text { T(Mp22, Mp 72) }
$$

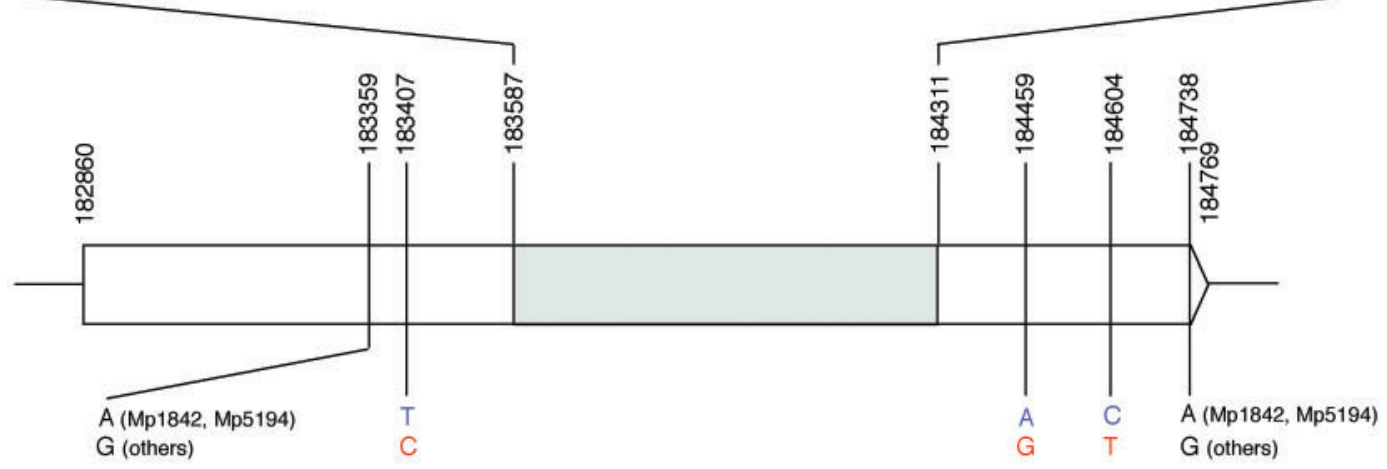

Subtype 1 variant (Mp4817)

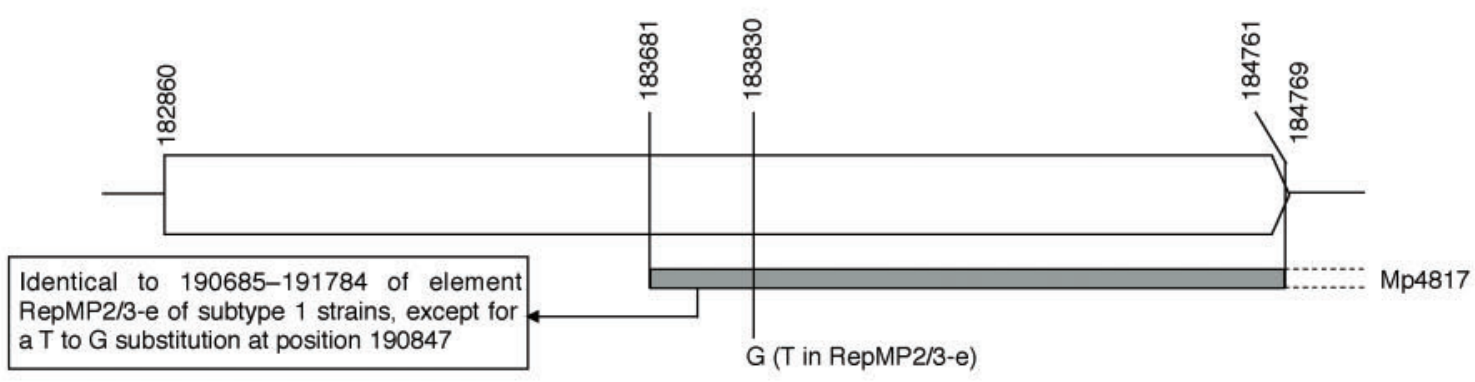

Subtype 2 variants (according to GenBank)

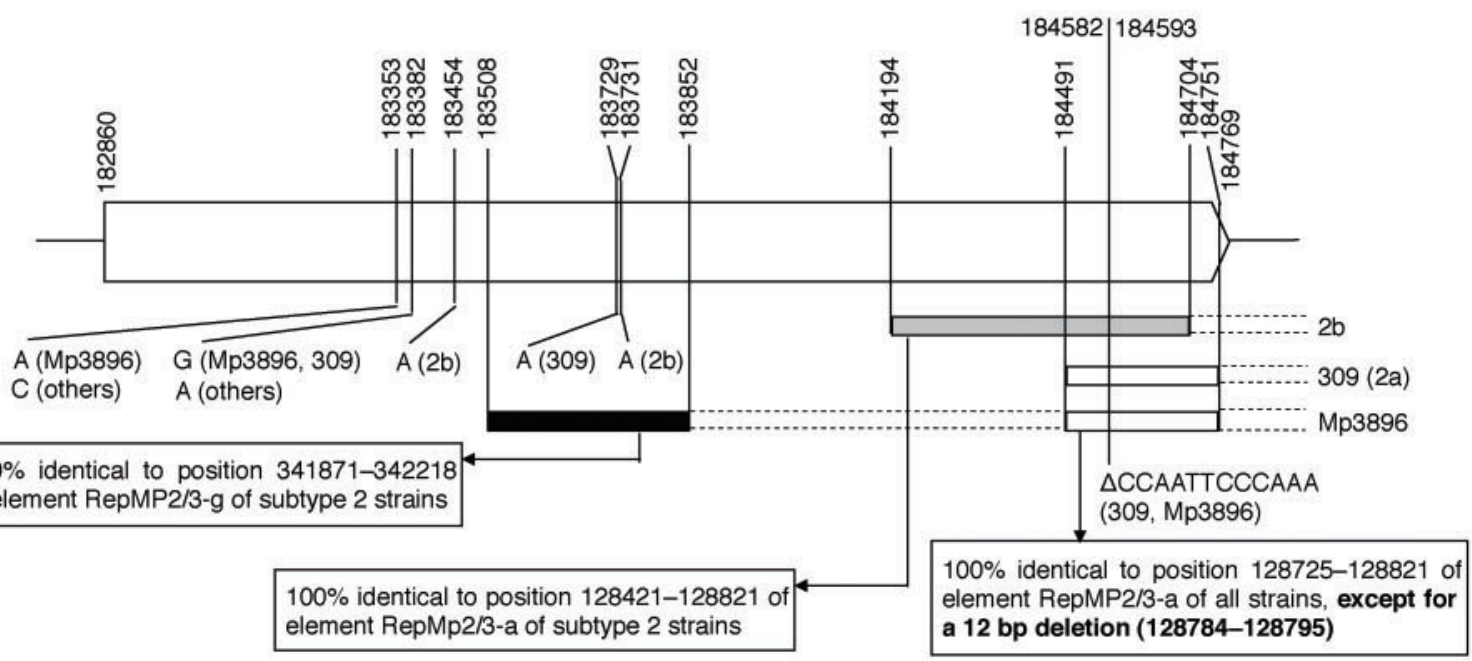


Fig. 3. Sequence variation of element RepMP2/3-d within M. pneumoniae strains. All nucleotide positions are taken from GenBank file U00089. Nucleotides, insertions and deletions $(\Delta)$ that are found in all subtype 1 and all subtype 2 strains are indicated in blue and red, respectively. The box at the top represents the sequence as found in most strains; the most variable part of this sequence is detailed in the inset at the top, in which the upper line shows the sequence of subtype 1 strains (1) and the lower line represents sequences of subtype 2 strains (2). The lower two boxes show the sequence organization as found in subtype 1 variant strain Mp4817 and in the subtype 2 variants (309, $2 \mathrm{~b}$ and Mp3896). The insets describe the putative origin of sequences that are not found in the RepMP2/3-d elements of most $M$. pneumoniae strains. The published sequence of the RepMP2/3-d element from strain MAC (GenBank accession no. AF290001) was found to contain several errors (as inferred from the sequences that we generated for $M A C$ as well as all other subtype 2 strains); these errors were found at positions 182872 ( $G$ should be A), 183622 (A should be $G$ ), 183631 ( $G$ should be A), 183727 (C should be T), 183728 (C should be G), 183730 ( $G$ should be $A$ ), 183746 ( $T$ should be $C$ ), 183842 (C should be $G$ ), 183848 ( $T$ should be $G$ ), 183855 ( $T$ should be $C$ ) and 183882 ( $G$ should be $T$ ). A single-nucleotide error was also found in the published sequence of element RepMP2/3-d of strain Mp1842 (GenBank accession no. AF290002): at position 182872, a G should be an A.

centred on the middle part of the element; these variations have previously been used to distinguish between subtype 1 and subtype 2 strains. Indeed, the vast majority of the variations found in this element are subtype-specific. Unlike the RepMP2/3 elements, the RepMP4 elements within the 23 strains do not show any indication of a previous inter-element recombination event. Nevertheless, the obtained sequences of the RepMP4 elements did allow us to explain the structure of the aberrant MPN141 gene within strain Mp3896, which previously could not be classified as either a subtype 1 or a subtype 2 strain (Pereyre et al., 2007). A region of 848 bp within the RepMP4-c element of Mp3896 was found to be identical to the sequence between positions 609478 and 610325 of the RepMP4-h element as found in all subtype 2 strains (Fig. $4 \mathrm{~h}$ and Fig. 5). Thus, it is likely that strain Mp3896 has acquired RepMP4-h sequences within its MPN141 gene by a recombination event in which the RepMP4-h element was used as the donor sequence. Taken together with the sequence data on the RepMP2/3 elements described above, the 'aberrant' strain Mp3896 can be described as a subtype 2 strain in which the MPN141 gene has undergone three different homologous recombination events, one involving its RepMP4 sequence and two involving its RepMP2/3 sequence.

\section{Classification of $M$. pneumoniae strains}

Taken together, our data indicate that M. pneumoniae strains can genetically and evolutionarily be divided into two lineages, i.e. a subtype 1 and a subtype 2 lineage. This notion was also supported by the analysis of phylogenetic trees that were generated with the RepMP2/3 and RepMP4 sequences. In each of these trees, a clear separation was observed between the subtype 1 and subtype 2 strains (data not shown).

The major variations in sequences within the MPN141 gene that have previously been detected in strains that were referred to as $2 \mathrm{a}, 2 \mathrm{~b}$ and variant 1 appear to be due solely to intra-genomic, inter-RepMP element recombination events. The configuration of the 'aberrant' MPN141 ORF structure of strain Mp3896 is also readily explained by such DNA rearrangements. Based on our results, a schematic representation was generated of the configuration of the RepMP $2 / 3$ and RepMP4 elements in each of the $M$. pneumoniae strains that have hitherto been analysed (Fig. 6). The variation in sequences of the RepMP elements that we have described allows for strains to be classified, analysed and named in a more comprehensive and systematic fashion than was previously possible. For instance, strain M129 may be classified as a strain of 'sequence type' $1-\mathrm{P} 1(4-\mathrm{c} ; 2 / 3-\mathrm{d})$, in which ' 1 ' indicates the subtype of the strain (in this case subtype 1 ) and $\mathrm{P} 1$ (4-c; $2 /$ 3-d) denotes the RepMP4 (4-c) and RepMP2/3 (2/3-d) organization within the P1 (MPN141) gene. Strain Mp4817 may be described as 1-P1(4-c; 2/3-d[183681] [190685$\left.\left.{ }_{191784} ; \mathrm{G}_{190847}\right] \mathrm{d}\left[{ }_{184761}\right]\right)$, in which 'd[183681]' and 'd[184761]' indicate the nucleotide positions of the RepMP2/3-d element between which sequences from an RepMP2/3-e element (e[190685-191784; $\left.\left.\mathrm{G}_{190847}\right]\right)$ have been inserted; ' $\mathrm{G}_{190847}$ ' indicates the presence of a $\mathrm{G}$ nucleotide (instead of a T nucleotide) at position 190847 of the inserted sequence. In short, strain Mp4817 may be referred to as a 1-P1(4-c; 2/3-d[e]d) strain. A short name may be particularly useful when different elements have donated sequences to a single RepMP element within the MPN141 gene. For example, strain Mp3896 may be classified in short as a 2-P1(4-c[h]c;2/3-d[g]d[a]d) strain. In Table 3, this proposed classification system of $M$. pneumoniae strains is applied to the strains listed in Fig. 6. Clear advantages of this method of classification are that: (i) it relies directly on DNA sequence information, and (ii) it is also applicable to newly identified strains possessing as yet unknown arrangements of RepMP sequences within their P1 gene. In the proposed classification system, the sequences of the RepMP elements outside of the P1 gene are not taken into account, because these elements are not likely to be expressed and therefore have no direct phenotypic consequence. 
(a)

RepMP4-a 130355-131994

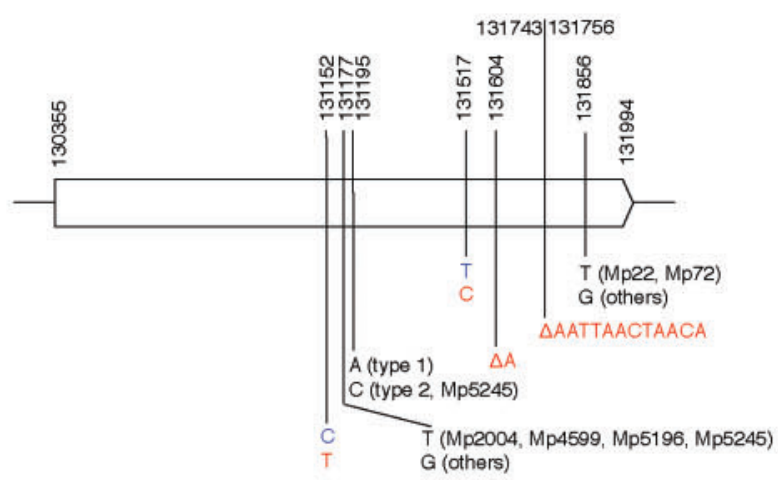

(c)

RepMP4-c 180820-182441

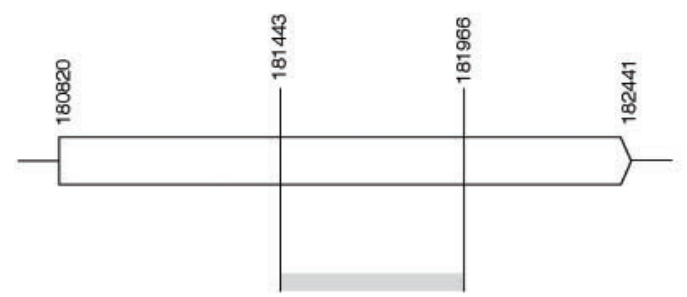

Variable region between subtype 1 and subtype 2 strains (see Fig. 5)

(e)

$$
\text { RepMP4-e }
$$

443048-444372

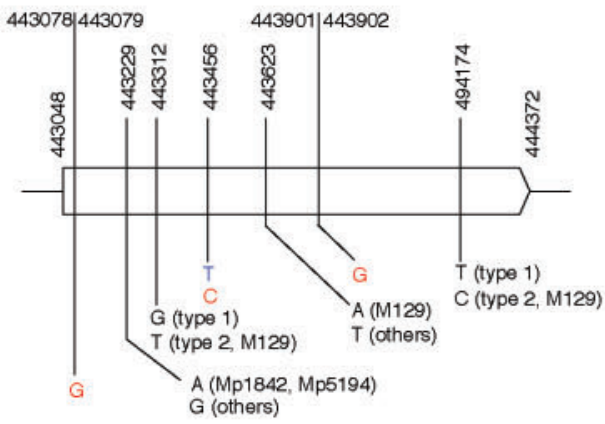

(g)

RepMP4-g

565050-566094

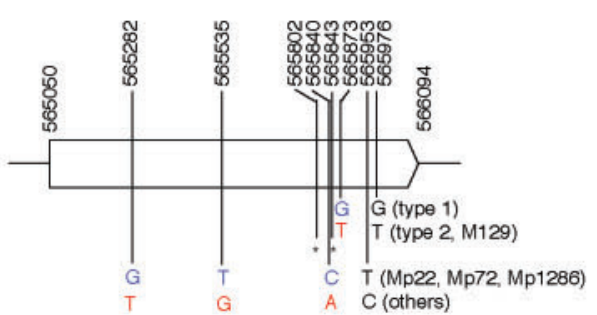

(b)

RepMP4-b

165770-167046

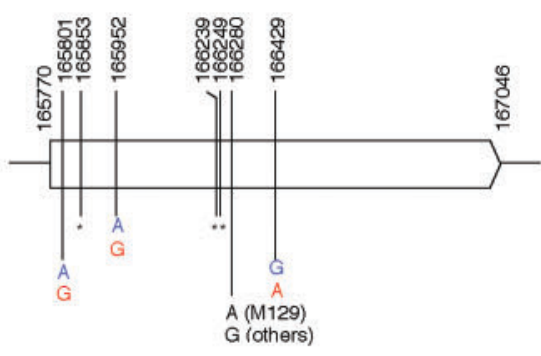

(d)

RepMP4-d 248448-249818

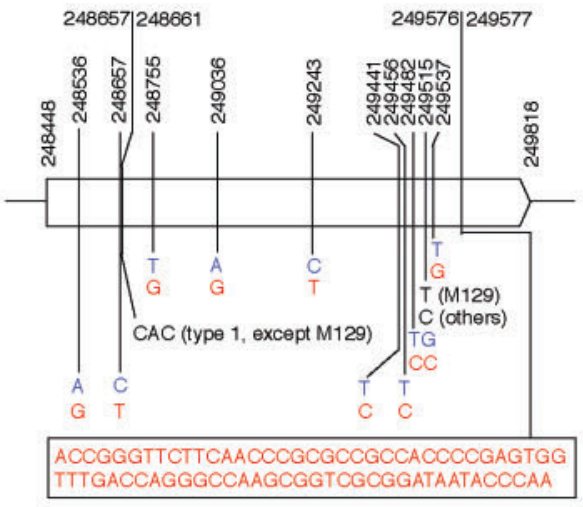

(f)

RepMP4-f

496473-497560

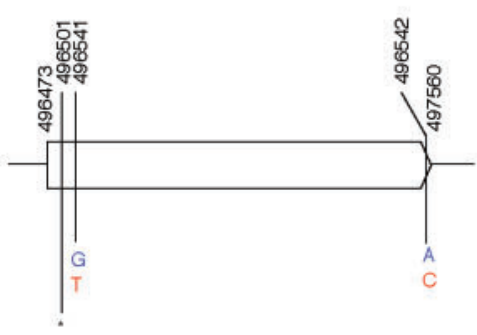

(h)

RepMP4-h

609094-610639

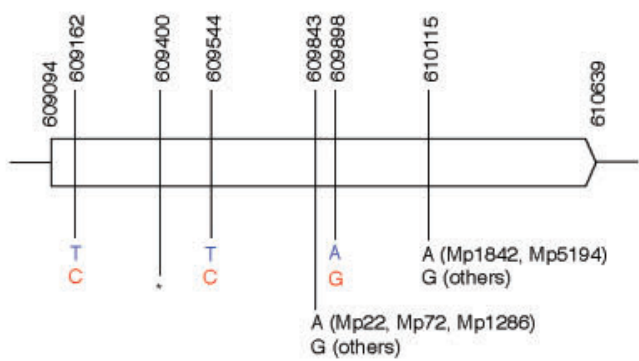


Fig. 4. Sequence variation among the eight RepMP4 elements within the $23 M$. pneumoniae strains. The sequences of the elements RepMP4-a to RepMP4-h are schematically represented in ( $a-h)$. The boxes indicate the length (drawn to scale) of each element and only list those nucleotide sequences within a given element that differ among the strains or between any strain and sequenced strain M129 (Himmelreich et al., 1996). Below the name of each element, its position within the genome of M129 is listed. The other positions in the figure also refer to this sequence. Nucleotides, insertions and deletions $(\Delta)$ that are found in all subtype 1 and all subtype 2 strains are indicated in blue and red, respectively. Sequence variations that are not subtype-specific are indicated in black. At some positions, indicated with an asterisk, the M129 sequence from GenBank differed from the sequences of M129 and all other strains as determined in this study. We therefore assume that the GenBank file is not correct at these positions. The incorrect nucleotides are: in RepMP4-b, a G at position 165853 (should be a C), a T at position 166239 (should be a C) and an A at position 166249 (should be a C); in RepMP4-f, a T at position 496501 (should be an A); in RepMP4-g, a G at position 565802 (should be a C) and a T at position 565843 (should be a G); in RepMP4-h, a T at position 609400 (should be a G). Because element RepMP4-c, which is localized within the P1 gene, is highly variable, the sequence variations within this element are depicted in a separate figure (Fig. 5).

\section{DISCUSSION}

The analysis of the DNA sequences of the RepMP2/3 and RepMP4 elements of various $M$. pneumoniae strains has revealed the following three major findings: (i) the genetic differences between subtype 1 and subtype 2 strains are reflected in each of the RepMP2/3 and RepMP4 elements within the $M$. pneumoniae genome, (ii) a similar homologous recombination event has occurred between two RepMP2/3 elements in four of the examined strains, and (iii) all previously described $\mathrm{P} 1$ genotypes can be explained by inter-RepMP recombination events.

\section{M. pneumoniae subtype 1 and 2 strains represent separate genetic lineages}

With regard to the first finding listed above, it is important to discriminate between the 'P1 genotype' and the 'subtype' of a strain. This is crucial because two M. pneumoniae isolates with a virtually identical genetic composition can have a significantly different $\mathrm{P} 1$ genotype as a consequence of a single recombination event between a RepMP element within the P1 gene and an element elsewhere in the genome. For instance, strain Mp4817 has previously been classified as an aberrant 'variant 1a' strain, because the DNA sequence of the RepMP2/3 element within its P1 gene ('P1 genotype') is considerably different from that of other strains (Dorigo-Zetsma et al., 2001). Due to the unavailability of the DNA sequences of other RepMP elements within this strain, the unusual structure of this RepMP element has even been considered to be the result of a combination of subtype 1 and subtype 2 sequences (Dorigo-Zetsma et al., 2001). By contrast, our data indicate that strain Mp4817 is a typical subtype 1 strain with respect to the sequences of all of its RepMP2/3 and RepMP4 elements. This strain does, however, differ from other subtype 1 strains because its RepMP2/3-e element has recombined with, and donated sequences to, the RepMP2/ 3-d element within the P1 gene. Similar considerations can be made for the other variant strains that have been isolated thus far, as summarized in Fig. 6. Thus, while strain Mp4817 should be classified as a subtype 1 strain, the variant $2 \mathrm{a}$ and $2 \mathrm{~b}$ strains, as well as strain Mp3896, should be classified as subtype 2 strains. Nevertheless, because these variant strains carry a P1 gene with a structure different from that of other subtype 2 strains, we have proposed an M. pneumoniae strain classification method in which subtype annotation is separated from the annotation of the P1 gene structure, as outlined in Table 3. Because the sequence data strongly indicate that subtype 1 and subtype 2 strains of M. pneumoniae represent separate and relatively conserved genetic entities, it is highly unlikely that isolates of a mixed subtype will be found in the near future.

In order to correctly type M. pneumoniae isolates, it seems sufficient to determine the sequences of the variable (RepMP) regions within the $\mathrm{P} 1$ gene and compare them with the sequences of the RepMP elements described in this study. Based upon the high level of sequence conservation that was found among the different RepMP elements within both subtype 1 and subtype 2 strains, we anticipate that the origins of 'novel' sequences within the P1 gene of new isolates can be traced back using the sequence library of RepMP elements that we have generated (Supplementary Table S2). Nevertheless, we cannot exclude the possibility that novel, deviant RepMP2/3- and RepMP4-like elements will be found in the future. It is also important to note that we did not thoroughly investigate the putative presence of RepMP elements at genome positions other than those known for reference strain M129.

\section{Homologous recombination between RepMP elements}

In four of the investigated strains, including three subtype 1 strains (Mp5, Mp72 and Mp2157) and one subtype 2 strain (Mp5181), sequences from element RepMP2/3-h appeared to be transferred to the RepMP2/3-c element. As the original sequence of element RepMP2/3-c seems to be 


\section{RepMP4-c \\ $180820-182441$}

181443 (1) CTACCGGTTTGCCGTTTTACTCGTGCCGCGCAGCGTGGTGTACTATGAGCAGTTGCAAAGGGGGTTGGGCTTIACCACAGCAGCGAACCGAGAGTGGTCAARATACTTCCACC---ACCGGGGCAATGTTTGGCTTGA (2) TTATCGGTTTGCCGTTTTACTCGTGCCGCGIAGCGTGGTGTACTATGAGCAGTTGCAGCGGGGGTTAGCGCTCCCTAACCAAGGGAGTTCGICAGGCTCAGACAGCACTAACCAAACAGGCGCAATGTTTGGCTTGA

181577 (1) AGGTGAAGAACGCCGAGGCGGACACCGCGAAG--AGCAATGNAAAACTCCA-GGGCGCTGAGGCCACTGGTTCTTCAACCACATCTGGATCTGGCCAATCCACCCAACGTGGGGTTCGTCAGGEGACACCAAAGTC (2) AGGTGAAGGATGCAACCGTGGATAGTTCGAAGCAATCAACGGAAAGCTTIAAAGGGCGAAGAATCGAGTTCCAGTTCCACCACATCT---TCCACCTCCACCACCCAACGTGGGGGTTCGTCAAATGAAAACAAAGTC G(Mp5181)

181711 (1) AAGGCTTTAAAAATAGAGGTGAAAAAGAAATC--GGACTCGGAG--_-_GACAATGGTCAGCTGCAGTTAGAAAAAAATGATCTCGCCAACGCTCCCATTAAGCGGAGCGAGGAGTCGGGTCAGTC (2) AAGGCGTTGCAGGTGGCGGTGAAAAAGAAATCCGGGAGTCAGGGCAACTCOGGTGACCAAGGCACCGAACAGGTGGAACTTGAATCTAATGATTTAGCCAACGCCCCGATTAAACGGGGCTCCAATAACAAOCAGCA

181830 (1) CGTCCAACTCAAGGCGGACGATTTTGGTACTGCCCTTTCCA.GTTCGGGATCAGGCGGCAACTCCAATCCCGGTTCCCCCACCCCCTGAAGGCCGTGGCTTGCGACTGAGCAAATTCACAAGGACCTCCCCAAATGAT (2) AGTCCAACTCAAGGCGGACGATTTTGGTACTGCCCCTTCCAGTTCGGGATCAGGCA--_--CCCAAGATGGCACCCCCACCCCCTGAACGCCGTGGTTAACGACTGAGCAAATTCACAACGACCCCGCCAAATTCG
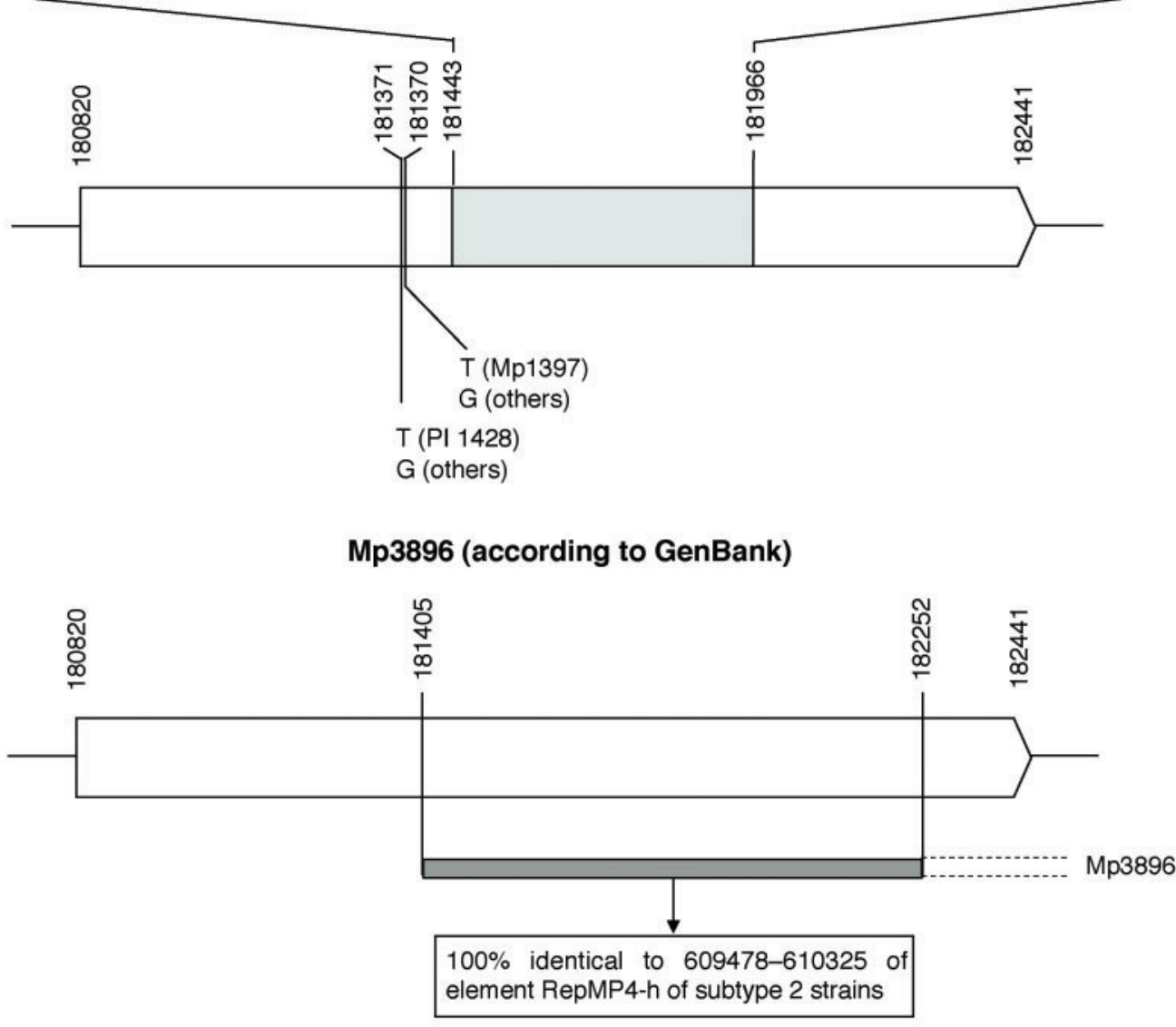

Fig. 5. Sequence variation of element RepMP4-c within $M$. pneumoniae strains. All nucleotide positions are taken from GenBank file U00089. Nucleotides, insertions and deletions $(\Delta)$ that are found in all subtype 1 and all subtype 2 strains are indicated in blue and red, respectively. The box at the top illustrates the sequence of most strains, whereas the box at the bottom describes the sequence of strain Mp3896. The inset at the bottom describes the putative origin of the sequence between positions 181405 and 182252 of the RepMP4-c element of this strain.

missing in these strains and the information from the RepMP2/3-h element is copied to the 'RepMP2/3-c location', we infer that these elements may have recombined by gene conversion. To our knowledge, this is the first time that putative homologous recombination events between RepMP2/3 elements outside of the P1 gene have been detected. As these DNA rearrangements do not include known operons or expressed genes, it is tempting to generalize that recombination among RepMP elements within the M. pneumoniae genome is governed solely by similarity in DNA sequence and is not executed in a directed fashion. Since a similar recombination event has occurred independently in four different strains, which represent $17 \%$ of all strains in this study, it is possible that this event is highly favourable, either because the local genomic structure surrounding elements RepMP2/3-c and RepMP2/3-h is 

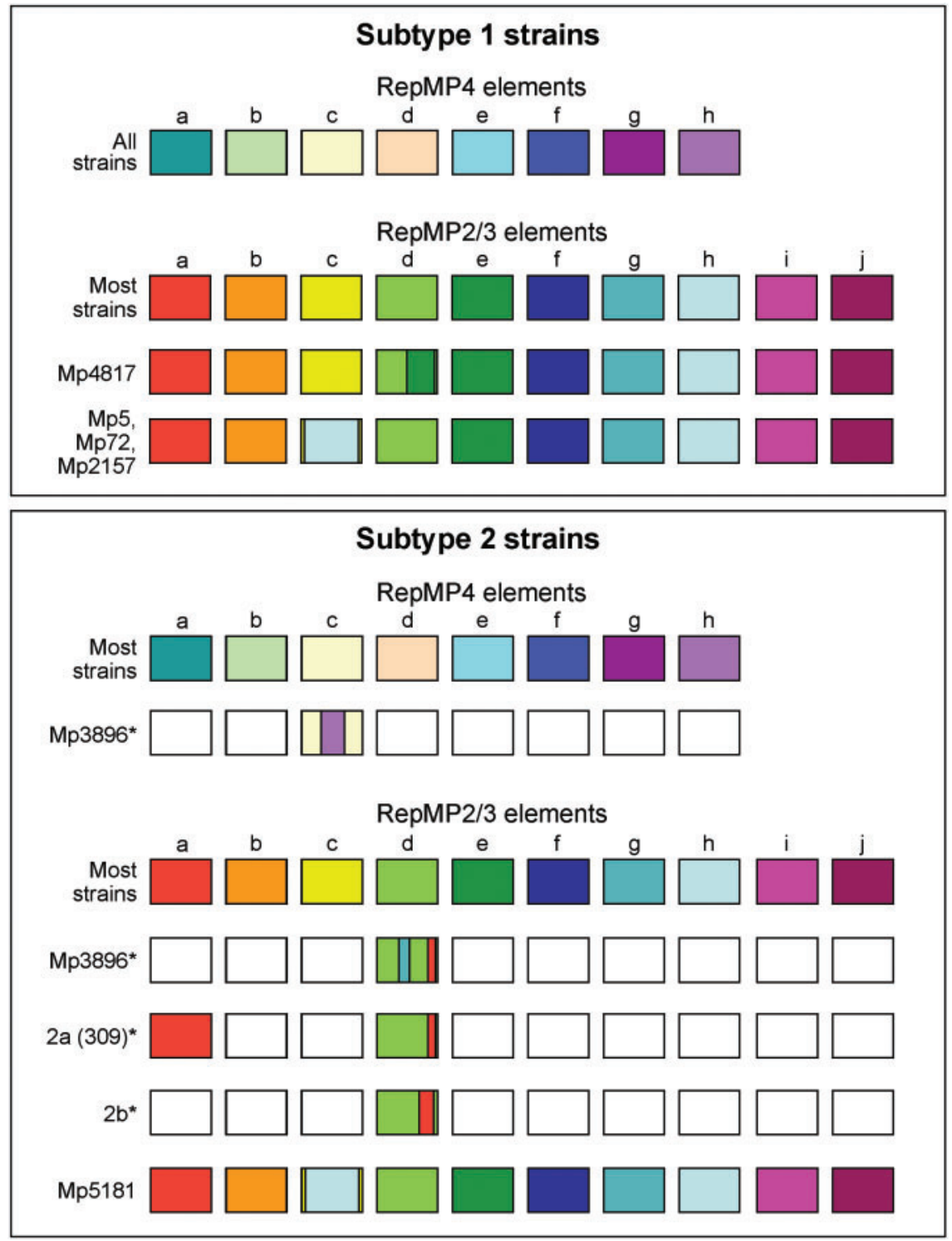

Fig. 6. Schematic representation of the configuration and structure of RepMP2/3 and RepMP4 elements in all M. pneumoniae strain sequences published to date. The top panel describes subtype 1 strains, whereas the lower panel describes subtype 2 strains. Each coloured block represents the sequence of a particular RepMP element, either RepMP4-a to - $h$ (at the top within each panel) or RepMP2/3-a to -j (at the bottom). The blocks are not drawn to scale. A colour change within a specific block indicates a putative DNA rearrangement event in which sequences from a specific element have been transferred to another element. For instance, within strain Mp4817, RepMP2/3-e sequences (in dark green) have been transferred to RepMP2/3-d (in light green). The colours of the elements labelled as 'All strains' or 'Most strains' were used as references. In the 'Subtype 1' panel, 'All strains' refers to all subtype 1 strains that have been examined in this study. 'Most strains' refers to all subtype 1 strains (top panel) or subtype 2 strains (bottom panel) that were studied, with the exception of the strains named separately in each panel. For the strains marked with an asterisk, i.e. strain 309 [2a (309)] (Kenri et al., 1999), the 2b strains (Dumke et al., 2006) and strain Mp3896 (Pereyre et al., 2007), the sequences of only a limited number of RepMP elements have been determined. These elements include those located within the P1 gene and, in the case of strain 309, also the RepMP2/3-a element. The elements that have not been sequenced have been left blank.

easily accessible for the enzymic recombination machinery or because these elements share a higher level of sequence similarity with each other than with any other RepMP2/3 element within the genome. However, there is no support for the latter notion, as all elements share significant similarity in at least part of their sequences (Supplementary Table S1). 
Table 3. Classification of M. pneumoniae strains on the basis of the origins of the RepMP sequences within the MPN141 or P1 gene

\begin{tabular}{|c|c|c|c|}
\hline \multirow{2}{*}{$\begin{array}{l}\text { Strain or } \\
\text { isolate }\end{array}$} & \multirow[t]{2}{*}{ Subtype ${ }^{\star}$} & \multicolumn{2}{|c|}{ Classification } \\
\hline & & Sequence type $\dagger$ & Short sequence type $\ddagger$ \\
\hline PI 1429 & 1 & $1-\mathrm{P} 1(4-\mathrm{c} ; 2 / 3-\mathrm{d})$ & $1-\mathrm{P} 1(4-\mathrm{c} ; 2 / 3-\mathrm{d})$ \\
\hline Mp4817 & 1 & 1-P1(4-c; $\left.2 / 3-\mathrm{d}\left[{ }_{183681}\right] \mathrm{e}\left[{ }_{190685-191784} ; \mathrm{G}_{190847}\right] \mathrm{d}\left[{ }_{184761}\right]\right)$ & $1-\mathrm{P} 1(4-\mathrm{c} ; 2 / 3-\mathrm{d}[\mathrm{e}] \mathrm{d})$ \\
\hline Mp2157§ & 1 & $1-\mathrm{P} 1(4-\mathrm{c} ; 2 / 3-\mathrm{d})$ & $1-\mathrm{P} 1(4-\mathrm{c} ; 2 / 3-\mathrm{d})$ \\
\hline MAC & 2 & $2-\mathrm{P} 1(4-\mathrm{c} ; 2 / 3-\mathrm{d})$ & $2-\mathrm{P} 1(4-\mathrm{c} ; 2 / 3-\mathrm{d})$ \\
\hline Mp3896 & 2 & $\begin{array}{c}2-\mathrm{P} 1\left(4-\mathrm{C}\left[{ }_{181405}\right] \mathrm{h}\left[{ }_{609478-610325}\right] \mathrm{C}\left[{ }_{182252}\right] ; 2 / 3-\mathrm{d}\left[{ }_{183508}\right] \mathrm{g}\left[{ }_{341871-}\right.\right. \\
\left.342218] \mathrm{d}\left[{ }_{183852-184491}\right] \mathrm{a}\left[{ }_{128725-128821 ; \Delta 128784-128795}\right] \mathrm{d}\left[{ }_{184751}\right]\right)\end{array}$ & $2-\mathrm{P} 1(4-\mathrm{c}[\mathrm{h}] \mathrm{c} ; 2 / 3-\mathrm{d}[\mathrm{g}] \mathrm{d}[\mathrm{a}] \mathrm{d})$ \\
\hline $309(2 a)$ & 2 & $2-\mathrm{P} 1\left(4-\mathrm{c} ; 2 / 3-\mathrm{d}\left[{ }_{184491}\right] \mathrm{a}\left[{ }_{128725-128821 ; \Delta 128784-128795}\right] \mathrm{d}\left[{ }_{184751}\right]\right)$ & $2-\mathrm{P} 1(4-\mathrm{c} ; 2 / 3-\mathrm{d}[\mathrm{a}] \mathrm{d})$ \\
\hline $2 b$ & 2 & $2-\mathrm{P} 1\left(4-\mathrm{c} ; 2 / 3-\mathrm{d}\left[{ }_{184194}\right] \mathrm{a}\left[{ }_{128421-128821}\right] \mathrm{d}\left[{ }_{184704}\right]\right)$ & $2-\mathrm{P} 1(4-\mathrm{c} ; 2 / 3-\mathrm{d}[\mathrm{a}] \mathrm{d})$ \\
\hline
\end{tabular}

*The subtype 1 and 2 annotation listed here is based on sequence differences found between subtype 1 and 2 strains in each RepMP2/3 and RepMP4 element.

$\dagger$ A sequence type is composed of a subtype number (either 1 or 2), followed by the arrangement of RepMP sequences (between brackets) within the P1 gene; ' 4 -c' refers to the RepMP4-c element and '2/3-d' refers to the RepMP2/3-d element. If these elements contain sequences derived from other RepMP elements, the nucleotide positions of the insertion sites are indicated between brackets; the inserted sequences are indicated by their positions within the RepMP element of origin (between brackets). In the sequence type of strain Mp4817, ' $\mathrm{G}_{190847}$ ' indicates that the nucleotide at position 190847 is replaced by a G nucleotide. The identity of the RepMP elements of origin are indicated by the letters ' $a$ ' to ' $j$ ' for RepMP2/3 and by 'a' to ' $h$ ' for RepMP4, as introduced in Table 2.

¥The short sequence types only list the origins of the RepMP sequences and not their full sequence details. Thus, elements with inserted sequences of different lengths, such as 309 and the $2 \mathrm{~b}$ strains, have the same name. The letters in parentheses refer to the origin of the inserted sequences; e.g. for strain Mp4817, '2/3-d[e]d' indicates that sequences from element RepMP2/3-e are inserted into RepMP2/3-d sequences.

\$Although these strains carry a recombined RepMP2/3 element (as summarized in Fig. 6), this element is not located within the P1 gene. Hence, these recombination events are not reflected in their 'sequence type'.

Gene conversion events were also hypothesized to have occurred in strain Mp4817 (described above) and strain 309, in which element RepMP2/3-d was found to carry sequences derived from element RepMP2/3-a (Kenri et al., 1999). Although the sequences of the RepMP elements outside of the P1 gene have not yet been determined for '2b' strains and strain Mp3896, it is likely that gene conversion-like events have also taken place in these strains. In more general terms, the current data on the dynamics of the exchange of sequence information among RepMP2/3 and RepMP4 elements suggests that these DNA rearrangements occur by (unidirectional) gene conversion and not by reciprocal exchange of homologous DNA sequences.

In conclusion, our data strongly support the hypothesis that the recombination of RepMP2/3 and RepMP4 elements forms the basis of antigenic variation of the P1 protein of M. pneumoniae (Dorigo-Zetsma et al., 2001; Kenri et al., 1999). The RepMP elements that are scattered around the bacterial genome appear to constitute a wealthy source for P1 sequence variability. It is therefore highly likely that novel variants of the $\mathrm{P} 1$ gene will continue to appear. This view stresses the importance of the availability of an adequate, universal typing method for M. pneumoniae strains as described in this study. It is clear, however, that the biological and evolutionary constraints on a recombination event within the $\mathrm{P} 1 \mathrm{ORF}$ are stringent, as the encoded P1 protein plays an essential role in the life cycle of $M$. pneumoniae. Each recombination event within the P1 ORF must therefore eventually result in the expression of a functional P1 protein. Nevertheless, we assume that recombination among RepMP elements within the P1 gene occurs far more frequently than would be deduced from the relatively infrequent detection of $\mathrm{P} 1$ sequence variation among either subtype 1 or subtype 2 strains. Clearly, recombination events leading to nonfunctional P1 proteins will have a biological disadvantage and not be selected for. The extent to which RepMP recombination occurs within the $M$. pneumoniae genome will therefore have to be studied in a context in which biological selection against 'unfavourable' DNA rearrangements is reduced to a minimum. We are currently exploring such contexts.

\section{REFERENCES}

Dallo, S. F., Horton, J. R., Su, C. J. \& Baseman, J. B. (1990). Restriction fragment length polymorphism in the cytadhesin P1 gene of human clinical isolates of Mycoplasma pneumoniae. Infect Immun 58, 2017-2020. 
Dandekar, T., Huynen, M., Regula, J. T., Ueberle, B., Zimmermann, C. U., Andrade, M. A., Doerks, T., Sanchez-Pulido, L., Snel, B. \& other authors (2000). Re-annotating the Mycoplasma pneumoniae genome sequence: adding value, function and reading frames. Nucleic Acids Res 28, 3278-3288.

Dorigo-Zetsma, J. W., Dankert, J. \& Zaat, S. A. (2000). Genotyping of Mycoplasma pneumoniae clinical isolates reveals eight P1 subtypes within two genomic groups. J Clin Microbiol 38, 965-970.

Dorigo-Zetsma, J. W., Wilbrink, B., Dankert, J. \& Zaat, S. A. (2001). Mycoplasma pneumoniae P1 type 1- and type 2-specific sequences within the P1 cytadhesin gene of individual strains. Infect Immun 69, $5612-5618$.

Dumke, R., Luck, P. C., Noppen, C., Schaefer, C., von Baum, H., Marre, R. \& Jacobs, E. (2006). Culture-independent molecular subtyping of Mycoplasma pneumoniae in clinical samples. J Clin Microbiol 44, 2567-2570.

Himmelreich, R., Hilbert, H., Plagens, H., Pirkl, E., Li, B. C. \& Herrmann, R. (1996). Complete sequence analysis of the genome of the bacterium Mycoplasma pneumoniae. Nucleic Acids Res 24, 44204449.

Hu, P. C., Huang, C. H., Collier, A. M. \& Clyde, W. A., Jr (1983). Demonstration of antibodies to Mycoplasma pneumoniae attachment protein in human sera and respiratory secretions. Infect Immun 41, 437-439.

Ingleston, S. M., Dickman, M. J., Grasby, J. A., Hornby, D. P., Sharples, G. J. \& Lloyd, R. G. (2002). Holliday junction binding and processing by the RuvA protein of Mycoplasma pneumoniae. Eur $J$ Biochem 269, 1525-1533.

Kenri, T., Taniguchi, R., Sasaki, Y., Okazaki, N., Narita, M., Izumikawa, K., Umetsu, M. \& Sasaki, T. (1999). Identification of a new variable sequence in the P1 cytadhesin gene of Mycoplasma pneumoniae: evidence for the generation of antigenic variation by DNA recombination between repetitive sequences. Infect Immun 67, 4557-4562.

Leith, D. K., Trevino, L. B., Tully, J. G., Senterfit, L. B. \& Baseman, J. B. (1983). Host discrimination of Mycoplasma pneumoniae proteinaceous immunogens. J Exp Med 157, 502-514.

Maniloff, J. (1992). Phylogeny of mycoplasmas. In Mycoplasmas: Molecular Biology and Pathogenesis, pp. 549-559. Edited by J.
Maniloff, R. N. McElhaney, L. R. Finch \& J. B. Baseman. Washington, DC: American Society for Microbiology.

Pereyre, S., Charron, A., Renaudin, H., Bebear, C. \& Bebear, C. M. (2007). First report of macrolide-resistant strains and description of a novel nucleotide sequence variation in the P1 adhesin gene in Mycoplasma pneumoniae clinical strains isolated in France over 12 years. J Clin Microbiol 45, 3534-3539.

Razin, S. \& Jacobs, E. (1992). Mycoplasma adhesion. J Gen Microbiol 138, 407-422.

Rocha, E. P. \& Blanchard, A. (2002). Genomic repeats, genome plasticity and the dynamics of Mycoplasma evolution. Nucleic Acids Res 30, 2031-2042.

Ruland, K., Wenzel, R. \& Herrmann, R. (1990). Analysis of three different repeated DNA elements present in the P1 operon of Mycoplasma pneumoniae: size, number and distribution on the genome. Nucleic Acids Res 18, 6311-6317.

Seto, S., Kenri, T., Tomiyama, T. \& Miyata, M. (2005). Involvement of P1 adhesin in gliding motility of Mycoplasma pneumoniae as revealed by the inhibitory effects of antibody under optimized gliding conditions. J Bacteriol 187, 1875-1877.

Sluijter, M., Hoogenboezem, T., Hartwig, N. G. \& Vink, C. (2008). The Mycoplasma pneumoniae MPN229 gene encodes a protein that selectively binds single-stranded DNA and stimulates recombinase A-mediated DNA strand exchange. BMC Microbiol 8, 167.

Su, C. J., Chavoya, A. \& Baseman, J. B. (1988). Regions of Mycoplasma pneumoniae cytadhesin P1 structural gene exist as multiple copies. Infect Immun 56, 3157-3161.

Su, C. J., Chavoya, A., Dallo, S. F. \& Baseman, J. B. (1990a). Sequence divergency of the cytadhesin gene of Mycoplasma pneumoniae. Infect Immun 58, 2669-2674.

Su, C. J., Dallo, S. F. \& Baseman, J. B. (1990b). Molecular distinctions among clinical isolates of Mycoplasma pneumoniae. J Clin Microbiol 28, 1538-1540.

Wilson, M. H. \& Collier, A. M. (1976). Ultrastructural study of Mycoplasma pneumoniae in organ culture. J Bacteriol 125, 332-339.

Edited by: J. Renaudin 